\title{
Unusual computed tomography findings of radionecrosis after chemoradiation of stage IV hypopharyngeal cancer: a case report
}

\author{
Yuh Baba ${ }^{1,2,3^{*}}$, Yasumasa Kato ${ }^{3}$, Kaoru Ogawa $^{2}$
}

\begin{abstract}
Introduction: Radionecrosis (post-radiotherapy laryngeal submucosal inflammation and necrosis) is a complication of (chemo) radiotherapy for hypopharyngeal cancer that is difficult to differentiate from tumor recurrence.

Case presentation: A 67-year-old Japanese man presented with a condition extremely difficult to diagnose differentially as radionecrosis or tumor recurrence after radiotherapy for hypopharyngeal cancer. Although tumor recurrence was suspected from clinical conditions and computed tomography findings, pathologic analysis revealed no evidence of tumor recurrence, and successful therapy with steroids and antibiotics reduced the mucosal edema.
\end{abstract}

Conclusion: Our findings emphasize the wide spectrum of radiographic presentation of radionecrosis after chemoradiation of stage IV hypopharyngeal cancer.

\section{Introduction}

Adverse reactions to radiotherapy can include both early and late complications. Late complications may arise more than several months after the completion of radiotherapy, even in patients who show no evidence of complications during exposure. These complications are generally irreversible, progress gradually, and cause crucial organ dysfunction. Late radiotherapy complications involving the head and neck area include disturbances of salivary secretion, jaw necrosis, chondronecrosis, hypothyroidism, subcutaneous tissue fibrosis, and development of second cancers. Improvements in radiotherapy seemed to have reduced the incidence of radiotherapy-related adverse reactions, from $5 \%$ during the 1970 s to $1 \%$ during the 1990 s $[1,2]$. Concomitant chemoradiation has become widely used in patients with advanced hypopharyngeal cancer, with an incidence of adverse reactions that has been predicted to increase in the future. To the best of our knowledge, however, few reports are available about computed tomography (CT) findings in patients with this condition $[3,4]$. We

\footnotetext{
* Correspondence: yuh_baba@hotmail.com

${ }^{1}$ Department of Otorhinolaryngology, Ohtawara Red Cross Hospital, 2-7-3

Sumiyoshi-cho, Ohtawara City, Tochigi 324-8686, Japan

Full list of author information is available at the end of the article
}

describe here a patient who experienced radionecrosis (post-radiotherapy laryngeal submucosal inflammation and necrosis) after chemoradiation of stage IV hypopharyngeal cancer, focusing on CT findings.

\section{Case presentation}

A 67-year-old Japanese man visited our department complaining of pharyngeal pain. He had no appreciable medical or family histories, had smoked 30 cigarettes per day for 35 years, and had no drinking history. The patient had become aware of pharyngeal pain and had visited his general practitioner, but his condition did not improve.

At his initial visit to our department, laryngopharyngeal fiberoptic endoscopy revealed a neoplastic lesion in the left pyriform recess (Figure 1a). Vocal cord fixation was also observed. Pathologic examination of a biopsy specimen revealed a poorly differentiated squamous cell carcinoma (SCC), and contrast CT showed a tumor occupying the left pharyngeal cavity and infiltrating the thyroid cartilage (Figure 1b). Lymph node metastasis in the left side of the neck was confirmed, but no distant metastases were found. The patient was diagnosed with stage IVA hypopharyngeal cancer (T4aN2bM0) and received two cycles of docetaxel, cisplatin, and 

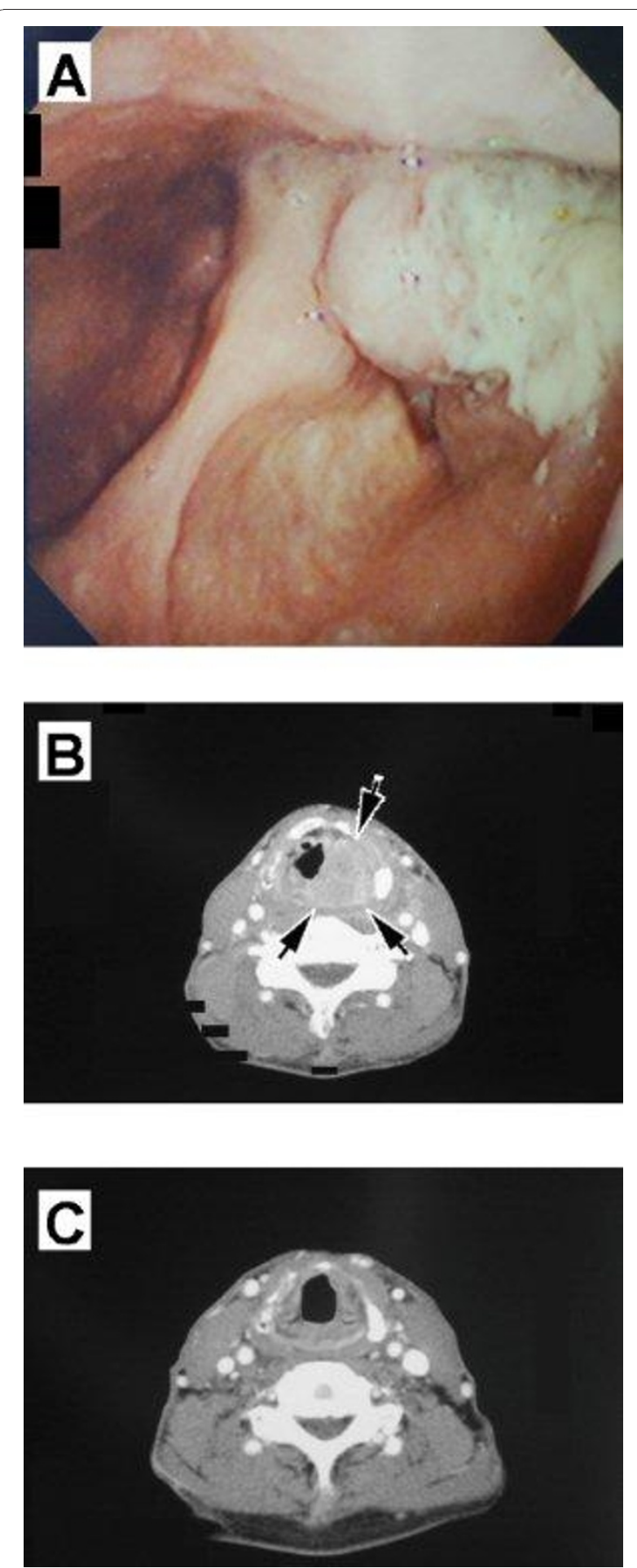

Figure 1 Before and after chemoradiotherapy. (a) Fiberoptic endoscopy showing a neoplastic lesion in the left pyriform recess. (b) CT showing a tumor occupying the left pharyngeal cavity and infiltrating the thyroid cartilage. (c) CT performed after the completion of chemoradiotherapy, showing no evidence of neoplastic lesions. 5-fluorouracil as neo-adjuvant chemotherapy. Three weeks after neo-adjuvant chemotherapy, he was started on concomitant chemoradiation, consisting of $10 \mathrm{mg} / \mathrm{m}^{2}$ docetaxel on days $1,8,15,22$, and 29 , and $3 \mathrm{D}$ radiotherapy, consisting of $2 \mathrm{~Gy}$ fractions once daily for five days per week, for a total dose of 62.0Gy in 31 fractions. The dose to the whole neck was 40.0Gy, and the dose to the primary site was $62.0 \mathrm{~Gy}$. Although the patient complained of mild pain in the throat, he was able to eat a sufficient number of light meals.

At approximately one month after completing radiotherapy, we took a biopsy sample of his left hypopharynx under direct laryngoscopy to evaluate response to therapy, as biopsy is done after initial treatment in the UMCC (University of Michigan Comprehensive Cancer Center) protocol 9520 [5]. Pathologic examination showed no obvious presence of remaining tumor, and CT showed no obvious neoplastic lesions (Figure 1c). The patient recovered his pretreatment vocal cord fixation and achieved a pathologic complete response (CR).

Later, as an outpatient, he gradually developed pharyngeal pain and dyspnea. Laryngopharyngeal fiberoptic endoscopy showed mucosal swelling in the pharynx and larynx. CT images of his neck, taken approximately eight months after the completion of radiotherapy, revealed a suggestive space-occupying lesion in the right lateral pharyngeal wall at the level of the epiglottis and a suggestive circumferential space-occupying lesion in the mucosa and airway constriction at the level of the thyroid cartilage. In detail, CT scans showed mixed masses of enhanced and non-enhanced lesions at both the epiglottis and thyroid-cartilage levels, strongly indicating tumor recurrence. Although we observed swelling of the surrounding soft tissues and segmentation or collapse of the thyroid cartilage, we did not observe defluxion of the arytenoid cartilage or abnormal gases contacting the cartilage (Figure 2a,b). Dyspnea was gradually aggravated, and the patient underwent a tracheostomy. Under general anesthesia, we removed biopsy samples from his lateral pharyngeal wall and larynx. Pathologic examination of these biopsy samples revealed no remaining cancer. Because the significant mucosal edema suggested the possibility of undetected submucosal recurrent lesions, we took additional biopsy samples under general anesthesia, but pathologic examination again showed no evidence of remaining cancer, although significant tissue degeneration and necrosis were observed. After a tentative diagnosis of radionecrosis after radiotherapy, the patient was treated with steroids and antibiotics. His laryngeal edema and swelling in the right lateral pharyngeal wall improved gradually, resulting in significant reductions in pharyngeal pain and pain during 


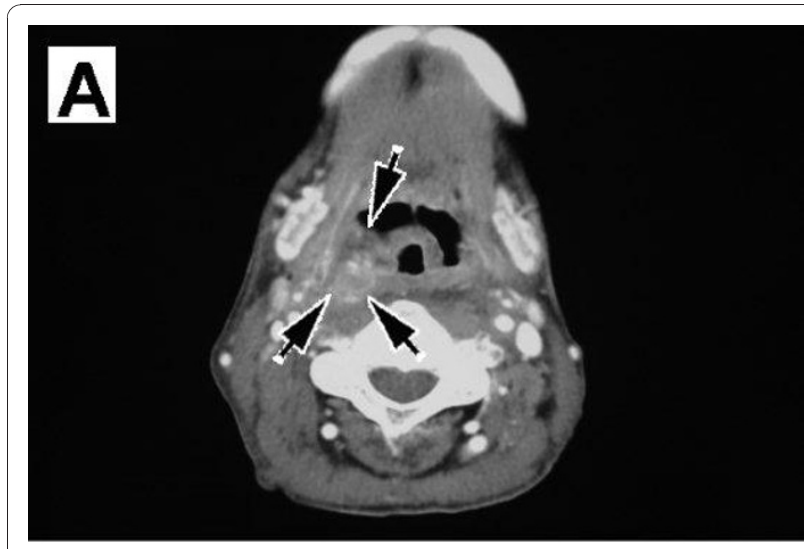

\section{B}

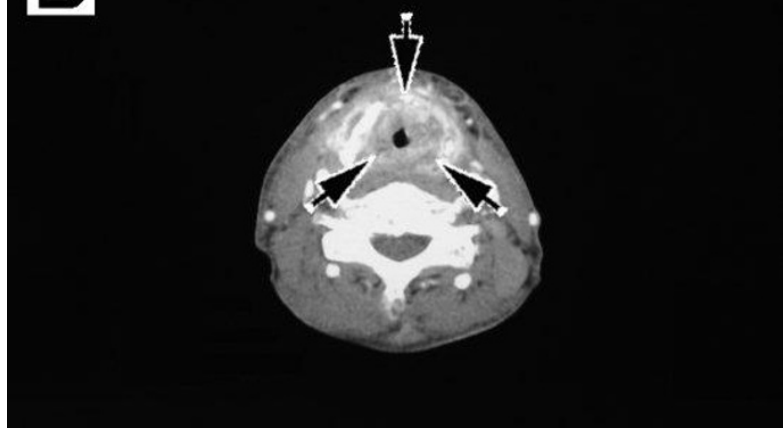

Figure 2 Follow-up CT results. (a) A suggestive space-occupying lesion in the right lateral pharyngeal wall at the level of the epiglottis, and (b) a suggestive circumferential space-occupying lesion in the mucosa and airway constriction at the level of the thyroid cartilage.

swallowing, allowing the patient to ingest solid foods. Moreover, the space-occupying lesion, which had been suspected as being tumor recurrence, had disappeared (Figure 3a,b). After CT evaluation, the tracheostomy was successfully reversed.

Two years after primary treatment with radiation, no evidence has been found of tumor recurrence. The patient's speech and deglutition were preserved, and his voice quality was within normal limits.

\section{Discussion}

Radionecrosis is one of the late complications of radiotherapy. Although its incidence is approximately $1 \%$ $[1,2]$, it is predicted to increase because of the increasing use of concomitant chemoradiotherapy. One possible mechanism of pathogenesis is vasculitis-induced narrowing and occlusion of the intravascular lumens [6]. Although radionecrosis usually occurs within the first year after radiotherapy [7], it may occur more than ten years later [8]. Treatment regimens for radionecrosis
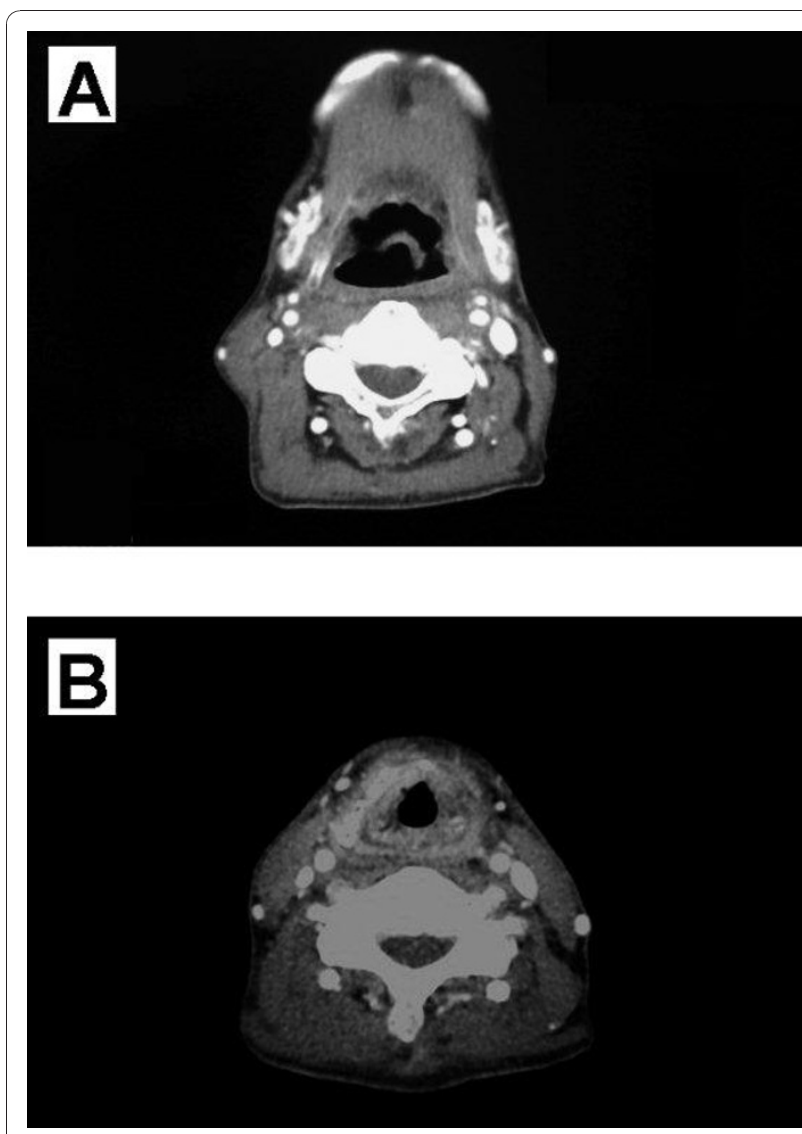

Figure 3 CT results after administration of steroids and antibiotics. These show the disappearance of space-occupying lesions initially suspected as tumor recurrence at the levels of the (a) epiglottis and (b) thyroid cartilage.

include application of antibiotics and steroids, hyperbaric oxygen therapy, and surgical intervention [9].

The clinical manifestations of radionecrosis, including hoarseness, difficulties with oral intake, pharyngeal pain, dyspnea, and skin fistula, are similar to those of tumor recurrence [10]. In addition, although radionecrosis is frequently accompanied by various CT findings, including swelling of the surrounding soft tissues, segmentation or collapse of the thyroid cartilage, defluxion of the arytenoid cartilage, and abnormal gases in contact with the cartilage, all of these findings are regarded as nonspecific $[3,4]$. Moreover, only about $50 \%$ of patients with radionecrosis present with these $\mathrm{CT}$ findings [11]. CT images of the neck of our patient revealed a suggestive space-occupying lesion in the right lateral pharyngeal wall at the level of the epiglottis and a suggestive circumferential space-occupying lesion in the mucosa and airway constriction at the level of the thyroid cartilage. In detail, $\mathrm{CT}$ scans showed mixed masses of enhanced and non-enhanced lesions at both the epiglottis and thyroid cartilage levels, masses that could have been 
easily mistaken for tumor recurrence. The enhanced lesions were likely viable infectious cells, consistent with the effectiveness of the antibiotics, whereas the nonenhanced lesions were likely necrotic cells.

In addition to the similarity of clinical conditions and CT findings in patients with radionecrosis and recurrent lesions, some submucosal recurrent lesions may not be detected by biopsy under direct laryngoscopy, especially in patients with significant mucosal edema. Moreover, in some patients, radionecrosis and recurrent tumor may occur concurrently. Thus, differential diagnosis between radionecrosis and tumor recurrence is considered extremely difficult. Positron emission tomography(PET)-CT is an alternative imaging modality that has been successfully used to differentiate postradiation changes, radionecrosis, and tumor recurrence. Furthermore, Fludeoxyglucose- (FDG)-PET scanning after radiation can predict tumor regrowth three months later [12]. However, follow-up PET-CT in our patient after therapy yielded negative results (data not shown).

Patients with T3/T4 hypopharyngeal SCC usually require total laryngopharyngectomy. Because our patient initially had bulky stage IV disease, associated with frank thyroid cartilage invasion, total laryngopharyngectomy was applicable. However, the patient desired organ preservation and therefore chose chemoradiotherapy as an alternative to total laryngopharyngectomy [5]. As a result, the patient retained appreciable vocal cord function of speech and deglutition, and his prognosis was good at two years after primary radiotherapy without tumor recurrence. The return of vocal cord function after chemoradiotherapy is a useful prognostic factor, with a fiveyear overall survival rate in patients with recovered vocal cord function of $100 \%$, compared with a two-year overall survival rate of patients with vocal cord fixation of less than $40 \%$ [13]. Our patient recovered vocal cord fixation, with no obvious signs of tumor recurrence more than two years after initial chemoradiotherapy. Therefore, chemoradiotherapy was appropriate for this patient.

\section{Conclusions}

A patient developed a condition after chemoradiotherapy for hypopharyngeal cancer that required differential diagnosis between radionecrosis and tumor recurrence. Our findings emphasize the wide spectrum of radiographic presentation of radionecrosis.

\section{Consent}

Written informed consent was obtained from the patient for publication of this case report and any accompanying images. A copy of the written consent is available for review by the Editor-in-Chief of this journal.

\section{Abbreviations}

CR: complete response; CT: computed tomography; SCC: squamous cell carcinoma.

\section{Author details}

'Department of Otorhinolaryngology, Ohtawara Red Cross Hospital, 2-7-3 Sumiyoshi-cho, Ohtawara City, Tochigi 324-8686, Japan. ${ }^{2}$ Department of Otorhinolaryngology, Head and Neck Surgery, Keio University, 35 Shinanomachi Shinjuku, Tokyo 160-0082, Japan. ${ }^{3}$ Department of Biochemistry \& Molecular Biology, Kanagawa Dental College, Yokosuka 238-8580, Japan.

\section{Authors' contributions}

All authors provided an equal intellectual contribution to this manuscript. All authors read and approved the final manuscript.

\section{Competing interests}

The authors declare that they have no competing interests.

Received: 2 April 2010 Accepted: 20 January 2011

Published: 20 January 2011

\section{References}

1. Oppenheimer RW, Krespi YP, Einhorn RK: Management of laryngeal radionecrosis: animal and clinical experience. Head Neck 1989, 11:252-256.

2. Stell PM, Morrison MD: Radiation necrosis of the larynx: etiology and management. Arch Otolaryngol 1973, 98:111-113.

3. Hermans R, Pameijer FA, Mancuso AA, Parsons JT, Mendenhall WM: CT findings in chondroradionecrosis of the larynx. AJNR Am J Neuroradiol 1998, 19:711-718.

4. Briggs RJ, Gallimore AP, Phelps PD, Howard DJ: Laryngeal imaging by computerized tomography and magnetic resonance following radiation therapy: a need for caution. J Laryngol Otol 1993, 107:565-568.

5. Worden FP, Moyer J, Lee JS, Taylor JM, Urba SG, Eisbruch A, Teknos TN, Chepeha DB, Prince ME, Hogikyan N, Lassig AA, Emerick K, Mukherji S, Hadjiski L, Tsien Cl, Miller TH, Wallace NE, Mason HL, Bradford CR, Wolf GT: Chemoselection as a strategy for organ preservation in patients with T4 laryngeal squamous cell carcinoma with cartilage invasion. Laryngoscope 2009, 119:1510-1517.

6. Filntisis GA, Moon RE, Kraft KL, Farmer JC, Scher RL, Piantadosi CA: Laryngeal radionecrosis and hyperbaric oxygen therapy: report of 18 cases and review of the literature. Ann Otol Rhinol Laryngol 2000, 109:554-562.

7. Keene M, Harwood AR, Bryce DP, van Nostrand AW: Histopathological study of radionecrosis in laryngeal carcinoma. Laryngoscope 1982, 92:173-180.

8. Shimizu N, Okamoto H, Fukuda T, Yane K, Hosoi H: Late laryngeal radionecrosis in severe arteriosclerosis. J Laryngol Otol 2005, 119:922-925.

9. Mcguirt WF, Greven KM, Keyes JW Jr, Williams DW, Watson N: Laryngeal radionecrosis versus recurrent cancer: a clinical approach. Ann Otol Rhinol Laryngol 1998, 107:293-296.

10. Chandler JR: Radiation fibrosis and necrosis of the larynx. Ann Otol Rhinol Laryngol 1979, 88:509-514.

11. Zbären P, Caversaccio M, Thoeny HC, Nuyens M, Curschmann J, Stauffer E: Radionecrosis or tumor recurrence after radiation of laryngeal and hypopharyngeal carcinomas. Otolaryngol Head Neck Surg 2006, 135:838-843.

12. Koike I, Ohmura M, Hata M, Takahashi N, Oka T, Ogino I, Lee J, Umezawa T, Kinbara $\mathrm{K}$, Watai $\mathrm{K}$, Ozawa $Y$, Inoue T: FDG-PET scanning after radiation can predict tumor regrowth three months later. Int I Radiat Oncol Biol Phys 2003, 57:1231-1238.

13. Solares CA, Wood B, Rodriguez CP, Lorenz RR, Scharpf J, Saxton J, Rybicki LA, Strome M, Esclamado R, Lavertu P, Adelstein DJ: Does vocal cord fixation preclude nonsurgical management of laryngeal cancer? Laryngoscope 2009, 119:1130-1134.

doi:10.1186/1752-1947-5-25

Cite this article as: Baba et al:: Unusual computed tomography findings of radionecrosis after chemoradiation of stage IV hypopharyngeal cancer: a case report. Journal of Medical Case Reports 2011 5:25. 